\title{
BMJ Open Prevalence and risk factors of latent tuberculosis infection in Africa: a systematic review and meta- analysis protocol
}

Tariro J Basera, ${ }^{1}$ Jabulani Ncayiyana, ${ }^{1}$ Mark E Engel ${ }^{2}$

To cite: Basera TJ, Ncayiyana J, Engel ME. Prevalence and risk factors of latent tuberculosis infection in Africa: a systematic review and metaanalysis protocol. BMJ Open 2017;7:e012636. doi:10.1136/ bmjopen-2016-012636

- Prepublication history and additional material are available. To view these files, please visit the journal online (http://dx.doi.org/ 10.1136/ bmjopen-2016-012636).

Received 13 May 2016 Revised 24 August 2016 Accepted 3 January 2017

\section{(a) CrossMark}

${ }^{1}$ Department of Epidemiology \& Biostatistics, School of Public Health, Faculty of Health Sciences, University of the Witwatersrand, Johannesburg, South Africa

${ }^{2}$ Department of Medicine, Faculty of Health Sciences University of Cape Town and Groote Schuur Hospital, Cape Town, South Africa

Correspondence to Jabulani Ncayiyana; jabulani. ncayiyana@wits.ac.za

\section{ABSTRACT}

Introduction Latent tuberculosis infection (LTBI) remains a major public health problem and one of the major contributors to the pool of active tuberculosis cases. The true burden of LTBI in Africa is not known. Early modelling studies estimate that over $33 \%$ of the world's population is infected with latent tuberculosis. We propose conducting a systematic review and a meta-analysis to evaluate the burden and risk factors of LTBI in Africa reported in studies from 2000 to 2017.

Methods and analysis We will include cross-sectional studies, cohort studies and case-control studies estimating either tuberculin skin test (TST) or interferon-gamma release assay (IGRA) confirmed prevalence of LTBI and associated risk factors among people in African countries. A comprehensive search of relevant literature will be conducted on electronic databases using common and medical subject heading (MeSH) terms for LTBI, and an African search filter. Risk of bias will be evaluated by assessing all qualifying full-text articles for quality and eligibility using a quality score assessment tool. Standardised data extraction will be carried out after which prevalence estimates will be pooled using randomeffects models in Stata V.13. Where there is sufficient data , subgroup meta-analyses will be conducted by risk factors including participant's age group, occupation, location and HIV status. This systematic review will be reported according to the Preferred Reporting Items for Systematic reviews and Meta-Analyses Protocols 2015 Statement. Ethics and dissemination No ethical issues were foreseen given that this was a protocol for a systematic review of published studies. The results of this study will be published in a peer-reviewed journal and presented at conferences.

Trial registration number Systematic review registration: PROSPERO CRD42016037997

\section{INTRODUCTION}

Tuberculosis (TB) is the second leading cause of mortality from an infectious disease globally after the HIV infection. ${ }^{1}$ In 2013, WHO estimated 9 million new TB cases and 1.5 million TB deaths globally, of which $80 \%$ of the cases and $70 \%$ of deaths were reported in low-income and middle-income countries. $^{2}$ Latent tuberculosis infection (LTBI)

\section{Strengths and Limitations of the study}

To our knowledge, this is the first systematic review protocol that has attempted to evaluate the burden of tuberculin skin test and interferon-gamma release assay-confirmed latent tuberculosis infection (LTBI) in Africa.

- This study could potentially inform policy and practice to reduce the reservoir of latently infected persons from which new tuberculosis (TB) cases arise.

- The chosen time period is short; however, it portrays an important era in Africa as significant gains have been made in the screening and treatment of TB, which could have theoretically huge impact on the burden of LTBI on the continent.

is defined as a state in which individuals harbour live Mycobacterium tuberculosis without evidence of manifestation of clinical or other symptoms of active disease. ${ }^{3} 4$ Projections from mathematical models in 2000 estimate that over $30 \%$ of the population globally were carriers of LTBI. ${ }^{5}$ Rates of infection with latent TB range from $31.2 \%$ in Ethiopia ${ }^{6}$ and $49 \%$ in Uganda $^{1}$ to $55.2 \%$ in South Africa. ${ }^{7}$ High prevalence of LTBI has been reported in at-risk populations such as miners $(89 \%),{ }^{8}$ and from $62 \%$ to $84 \%$ in healthcare workers in high incidence countries. ${ }^{9} 10$ A significant number of active TB cases arise from people with LTBI within a period of $2-5$ years following primary infection. ${ }^{4}$ Between $5 \%$ and $15 \%$ of the people with LTBI progress to active TB and the risk of active TB increases with poor immunity $(30 \%$ among those infected with HIV). ${ }^{11} 12$

The pathogenic state of bacterial infection and probability of reactivation depend on the balance between host immunity and the influence of exogenous factors. The following factors substantially increase the likelihood of progression of latent infection: 
suppression of cellular immunity by HIV infection, HIV immunosuppression, ${ }^{11}$ glucocorticoids, ${ }^{12}$ blood or organ transplant ${ }^{1314}$ and tumour necrosis factor $\alpha$ inhibitors. ${ }^{15}$ Other factors associated with LTBI include age, positive HIV status, working as physicians/nurses or miners, diabetes and malnutrition. ${ }^{17-9}$

Currently, it is not possible to directly identify LTBI in humans. ${ }^{3} 11$ LTBI is diagnosed by detecting memory T-cell response against latent infection with $M$. tuberculosis with the use of tuberculin skin test (TST) or interferon-gamma release assays (IGRAs) ${ }^{16}$ Thus, it is imperative to develop tools to improve the diagnostic capacity of current methods. Although currently no standard immunodiagnostic biomarkers have been identified to measure LTBI, there is growing landscape of chemokines, tumour necrosis factor, interleukin growth factors and soluble receptors under development that could improve diagnostic capacity. ${ }^{17}$

TST is sensitive, inexpensive and widely used particularly in low-resource settings including sub-Saharan Africa. ${ }^{6}$ TST has low sensitivity among people with suppressed immunity and it has low specificity in predicting reactivation TB among people vaccinated with Bacillus Calmette-Guérin (BCG) and it is vulnerable to react to environmental non-TB Mycobacterium. ${ }^{16}{ }^{18}$ Conventional studies on prevalence of LTBI used the TST and were thus hampered by the low specificity of the TST and its cross-reactivity with BCG and exposure to environmental mycobacteria, hence increasing the risk of overestimating LTBI. ${ }^{19}$ IGRAs has high specificity compared with TST because the former measure cellular response of T-lymphocytes to antigens of M. tuberculosis found in BCG and most non-TB mycobacteria. ${ }^{11}$ However, recent studies involving serially tested healthcare workers in the USA have shown that false conversions (from a negative to a false positive result) and reversions (from a positive to a false negative result) are more common with IGRAs than with TSTs. ${ }^{18}$ In areas with high TB prevalence, the sensitivity of IGRAs has not shown superiority over the conventional TST. ${ }^{20}$

We therefore propose to conduct a systematic review and meta-analysis to evaluate the burden of TST and IGRA-confirmed LTBI and associated risk factors in Africa.

\section{OBJECTIVES}

The objective of this review is to conduct a systematic review and meta-analysis of studies assessing the prevalence and risk factors of TST and IGRA-confirmed LTBI among people in African countries.

\section{REVIEW QUESTION}

This systematic review has been guided by the following research question: What is the prevalence of TST and IGRA-confirmed LTBI in African countries as reported in studies from 2000 to 2017 ?

\begin{tabular}{ll}
\hline Table 1 & Search strategy \\
\hline Search & $\begin{array}{l}\text { MeSH term (modified as needed for use in } \\
\text { other databases) }\end{array}$ \\
\hline$\# 1$ & Prevalence \\
$\# 2$ & frequency \\
$\# 3$ & rate \\
$\# 4$ & proportion \\
$\#$ \#6 & epidemiology \\
$\# 7$ & statistic \\
\hline$\# 1$ & LTBI \\
\hline$\# 9$ & Latent tuberculosis infection \\
\hline 10 & Latent mycobacterium tuberculosis \\
$\# 11$ & Mycobacterium tuberculosis \\
$\# 12$ & TST \\
$\# 13$ & Tuberculin skin test \\
$\# 14$ & Tuberculin test positivity \\
$\# 15$ & Interferon-gamma release assay test \\
$\# 16$ & Interferon gamma test positive \\
$\# 17$ & \#8 OR \#9 OR \#10 OR \#11 OR \#12 OR \#13 OR \#14 \\
\hline OR \#15 OR \#16 \\
\hline 18 & African Search Filter (see online Appendix) \\
\hline 19 & \#7 AND \#17 AND \#18 \\
\hline &
\end{tabular}

\section{METHODS}

\section{Criteria for considering studies for the review}

Inclusion criteria

1. Studies describing the prevalence of LTBI across all age groups, resident in countries belonging to the African continent, in the geographic regions of subSaharan and North Africa diagnosed with either TST or IGRA-confirmed M. tuberculosis from all ethnicities, socioeconomic and educational backgrounds.

2. Cross-sectional, cohort and case-control studies will be included. For the purpose of this review, the diagnosis of LTBI was determined by TST or IGRA.

3. Published articles, thesis, bulletins, reports and conference proceedings will be considered. Articles published in any language, with full English abstracts are eligible for inclusion.

\section{Exclusion criteria}

1. Narrative reviews, opinion pieces and letters or any other publications lacking primary data and/or explicit descriptions of the method.

2. Studies deemed to have a low-quality score in the assessment of risk of bias (ie, $\leq 5$ using the Hoy scale).$^{21}$

\section{Search strategy to identify relevant studies}

To maximise sensitivity, a broad search strategy was designed as shown in table 1 . Medical subject heading $(\mathrm{MeSH})$ terms for LTBI will be used in the main search 
combined with an African search filter developed by Pienaar et $\mathrm{al}^{21}$ and Eisinga et $\mathrm{al}^{22}$ to identify prevalence studies conducted from January 2000 to the African filter comprising country names as well as truncated terms such as 'east* Africa' to ensure that records indexed using regional, rather than country-specific terms, were included. The African search filter also includes the English name as well as the name of the country in the language relevant to that region. We will search for relevant articles in the following databases: PubMed, Web of Science, Africa-Wide: NiPAD, Scopus and WHOLIS.

In an attempt to identify all relevant articles, the initial search will not restricted by age or language of publication or publication type. The authors will then independently analyse the text words contained in the title and abstract, and the index terms used to describe the article. Potentially relevant thesis, bulletins, conference proceedings and reports will also be screened, including ones from WHO. Additional publications will be identified from references cited in relevant articles and searches in Google Scholar. Articles will be restricted to publications between 2000 and 2017, and the included studies will not be restricted by language.

\section{Selecting studies for inclusion}

Following scrutiny of titles and abstracts, full-text articles will be retrieved for studies meeting with the inclusion criteria. Two authors will independently evaluate and appraise the results of the searches, and studies will then be marked as 1) included, 2) excluded or 3) or marked as pending if the reviewer was uncertain. The independent evaluations will then be compared thereafter and discrepancies will be resolved by consensus.

If necessary, a third reviewer will act as an arbitrator. A flow chart will be produced to facilitate transparency of the selection process.

\section{Quality appraisal of included studies}

A Quality Index based on existing indices will be used to rate the methodological parameters of studies meeting the inclusion criteria. The following items are captured by the eight item index: sampling, diagnostic heterogeneity, followup rates and diagnostic assessment. A total quality score will be derived from summing the individual item scores and it ranges from 0 (lowest) to 16 (highest). The scores will be calculated and documented during the data extraction process. Study quality will be assessed using a quality assessment tool modified by Hoy $e t a l^{23}$ and as used by Barth $e t a l^{24}$ (table 2). Based on this tool, studies are rated as low risk, moderate risk and high risk for scores $\leq 5,6-8$ and $>8$, respectively. Discrepancies will be discussed and resolved by consensus between the authors and an independent reviewer. An evaluation of the risk of bias allows for sensitivity analysis.

\section{Data extraction and management}

The process of selecting articles for inclusion is managed by importing articles into EndNote $\mathrm{X} 7$ software. Two
Table 2 The quality assessment criteria for prevalence studies $^{23}$

\begin{tabular}{ll}
\hline External validity & Score \\
\hline 1. Was the study's target population & (one point) \\
a close representation of the national & \\
population in relation to relevant & \\
variables? &
\end{tabular}

2. Was the sampling frame a true or close (one point) representation of the target population?

3. Was some form of random selection (one point) used to select the sample, or was a census undertaken?

4. Was the likelihood of non-response bias (one point) minimal?

\begin{tabular}{|c|c|}
\hline Total & (four points) \\
\hline Internal validity & Score \\
\hline $\begin{array}{l}\text { 1. Were data collected directly from the } \\
\text { subjects (as opposed to a proxy)? }\end{array}$ & (one point) \\
\hline $\begin{array}{l}\text { 2. Was an acceptable case definition used } \\
\text { in the study? }\end{array}$ & (one point) \\
\hline $\begin{array}{l}\text { 3. Was the study instrument that } \\
\text { measured the parameter of interest shown } \\
\text { to have validity and reliability? }\end{array}$ & (one point) \\
\hline $\begin{array}{l}\text { 4. Was the same mode of data collection } \\
\text { used for all subjects? }\end{array}$ & (one point) \\
\hline $\begin{array}{l}\text { 5. Was the length of the shortest } \\
\text { prevalence period for the parameter of } \\
\text { interest appropriate? }\end{array}$ & (one point) \\
\hline $\begin{array}{l}\text { 6. Were the numerator(s) and } \\
\text { denominator(s) for the parameter of } \\
\text { interest appropriate? }\end{array}$ & (one point) \\
\hline Total & (six points) \\
\hline
\end{tabular}

independent reviewers will extract relevant data. Fields include study descriptors (authors, publication year, research design and length of follow-up), key study measures and outcomes (diagnostic inclusion criteria and rates) and, study entry restrictions, gender and age distribution. Potential caveats of relevant studies, particularly with regard to possible bias introduced with the study, will be noted.

\section{Data synthesis and assessment of heterogeneity}

Quantitative data synthesis includes two steps namely, the identification of data sources and documenting numerators and denominators that were used for prevalence calculations and second, the application of the Freeman-Tukey double arcsine transformation to stabilise the variance of study-specific prevalence. This serves to minimise the influence from studies with extremely small or extremely large prevalence estimates before pooling data using the random-effects meta-analysis. ${ }^{25}$ For each study, the reported prevalence will be recalculated to confirm numerators and denominators and, if necessary, adjustments will be made. A random-effects meta-analysis 
model using the 'metaprop' routine in Stata V.13 will be performed to pool prevalence estimates.

The second step also involves calculating the overall pooled estimate as well as the $95 \% \mathrm{CI}$ in order to account for variability between studies. Where possible, a trend analysis is performed to determine trends of LTBI. Standard errors will be derived from previous studies, which presented the corresponding numerator and denominator for prevalence estimates of LTBI.

Heterogeneity from the studies included will be assessed using the $\mathrm{I}^{2}$ statistic, which will be reported as a percentage in order to establish the degree of variation between the studies. ${ }^{26}$ The categories of heterogeneity are defined as follows: $\geq 76 \%-100 \%$ considerable, $51 \%-75 \%$ substantial, $26 \%-50 \%$ moderate and $25 \%$ as low heterogeneity. To further identify heterogeneity, we will use the $\chi^{2}$ test (with significance defined at the alpha-level of $10 \%$ ) and non-overlapping CIs as an indicator of statistically significant differences between studies. Should significant inconsistency between studies be found, sensitivity analysis will be performed to ascertain the sources of heterogeneity.

In addition, we will perform subgroup analyses and the findings will be narratively explained together with tables and figures where applicable. Any discrepancies or disagreements will be documented and discussed with a third author.

\section{Assessment of reporting biases}

Publication bias will be assessed using symmetry of funnel plots if 10 or more eligible studies are identified.

\section{Reporting of this review}

The eligibility criteria of studies and the selection process of relevant articles will be summarised as flow diagrams. This systematic review will be reported according to the Preferred Reporting Items for Systematic reviews and Meta-analysis guidelines. ${ }^{27}$

The primary outcome of this systematic review is to determine the prevalence of TST and IGRA-confirmed LTBI in Africa.

The secondary outcomes include examining the quality of the studies mentioned in this review, assessing trends, demographic characteristics and risk factors of TST and IGRA-confirmed LTBI in African countries.

\section{Ethics and dissemination}

No formal ethical review was required as the systematic reviews will use publicly available data. The findings of this systematic review will be disseminated through peer-reviewed journal publications and conference proceedings. To our knowledge, there are no systematic reviews that have specifically looked at the prevalence of TST and IGRA-confirmed LTBI in Africa.

We believe that the findings of this systematic review will have implications for policy, practice and development of diagnostic tools for LTBI, informed by data solely from Africa where the burden of TB is among the greatest.
Contributors TJB wrote the first draft and all authors edited the subsequent versions of the draft. TJB and JN developed the protocol, conducted the searches and extracted the data. MEE oversaw the final analysis of the data. All authors have reviewed and accepted the final version of the protocol and have given their permission for publication.

Competing interests None declared.

Provenance and peer review Not commissioned; externally peer reviewed.

Open Access This is an Open Access article distributed in accordance with the Creative Commons Attribution Non Commercial (CC BY-NC 4.0) license, which permits others to distribute, remix, adapt, build upon this work non-commercially, and license their derivative works on different terms, provided the original work is properly cited and the use is non-commercial. See: http://creativecommons.org/ licenses/by-nc/4.0/

(C) Article author(s) (or their employer(s) unless otherwise stated in the text of the article) 2017. All rights reserved. No commercial use is permitted unless otherwise expressly granted.

\section{REFERENCES}

1. Kizza FN, List J, Nkwata AK, et al. Prevalence of latent tuberculosis infection and associated risk factors in an urban African setting. BMC Infect Dis 2015;15:165-73.

2. World Health Organization. Global tuberculosis Report 2014. Geneva, Switzerland: WHO Library, 2014.

3. Mack U, Migliori GB, Sester M, et al. LTBI: latent tuberculosis infection or lasting immune responses to $M$. tuberculosis? A TBNET consensus statement. Eur Respir J 2009;33:956-73.

4. Sharma SK, Mohanan S, Sharma A. Relevance of latent TB infection in areas of high TB prevalence. Chest 2012;142:761-73.

5. Corbett EL, Watt CJ, Walker N, et al. The growing burden of tuberculosis: global trends and interactions with the HIV epidemic. Arch Intern Med 2003;163:1009-21.

6. Legesse M, Ameni G, Mamo G, et al. Community-based crosssectional survey of latent tuberculosis infection in Afar pastoralists, Ethiopia, using QuantiFERON-TB Gold In-Tube and tuberculin skin test. BMC Infect Dis 2011;11:89-97.

7. Mahomed H, Hawkridge T, Verver S, et al. Predictive factors for latent tuberculosis infection among adolescents in a high-burden area in South Africa. Int J Tuberc Lung Dis 2011;15:331-6.

8. Hanifa Y, Grant AD, Lewis J, et al. Prevalence of latent tuberculosis infection among gold miners in South Africa. Int J Tuberc Lung Dis 2009;13:39-46.

9. Rutanga C, Lowrance DW, Oeltmann JE, et al. Latent tuberculosis infection and associated factors among Health Care Workers in Kigali, Rwanda. PLoS One 2015;10:e0124485.

10. Adams S, Ehrlich R, Baatjies R, et al. Incidence of occupational latent tuberculosis infection in south African healthcare workers. Eur Respir J 2015;45:1364-73.

11. Getahun $H$, Matteelli $A$, Chaisson RE, et al. Latent mycobacterium tuberculosis infection. N Engl J Med 2015;372:2127-35.

12. World Health Organization. Global tuberculosis Report 2012. Geneva, Switzerland: WHO Library, 2013.

13. World Health Organization. Global tuberculosis Report 2014. Geneva, Switzerland: WHO Library, 2014.

14. Sidhu A, Verma G, Humar A, et al. Outcome of latent tuberculosis infection in solid organ transplant recipients over a 10-year period. Transplantation 2014;98:671-5.

15. Al-Anazi KA, Al-Jasser AM, Alsaleh K. Infections caused by Mycobacterium tuberculosis in recipients of hematopoietic stem cell transplantation. Front Oncol 2014;4:231.

16. Keane J, Bresnihan B. Tuberculosis reactivation during immunosuppressive therapy in rheumatic diseases: diagnostic and therapeutic strategies. Curr Opin Rheumatol 2008;20:443-9.

17. O'Garra A, Redford PS, McNab FW, et al. The immune response in tuberculosis. Annu Rev Immunol 2013;31:475-527.

18. Chegou NN, Heyckendorf J, Walzl G, et al. Beyond the IFN- $\gamma$ horizon: biomarkers for immunodiagnosis of infection with Mycobacterium tuberculosis. Eur Respir J 2014;43:1472-86.

19. Dorman SE, Belknap R, Graviss EA, et al. Interferon- $\gamma$ release assays and tuberculin skin testing for diagnosis of latent tuberculosis infection in healthcare workers in the United States. Am J Respir Crit Care Med 2014;189:77-87.

20. Schablon A, Beckmann G, Harling M, et al. Prevalence of latent tuberculosis infection among health care workers in a hospital for pulmonary diseases. J Occup Med Toxicol 2009;4:1. 
21. Pienaar E, Grobler L, Busgeeth $\mathrm{K}$, et al. Developing a geographic search filter to identify randomised controlled trials in Africa: finding the optimal balance between sensitivity and precision. Health Info Libr J 2011;28:210-5.

22. Eisinga A, Siegfried N, Clarke M. Sensitivity and precision of terms in phases I, II and III of the Cochrane Highly sensitive search strategy (HSSS) for identifying reports of randomized trials in Africa in HIV/AIDS in MEDLINE. XIV Cochrane Colloquium 2006.

23. Hoy D, Brooks P, Woolf A, et al. Assessing risk of Bias in prevalence studies: modification of an existing tool and evidence of interrater agreement. J Clin Epidemiol 2012;65:934-9.
24. Barth DD, Mayosi BM, Jabar A, et al. Prevalence of group A streptococcal disease in North and Sub-Saharan Africa: a systematic review protocol. BMJ Open 2015;5:e008646.

25. Barendregt JJ, Doi SA, Lee YY, et al. Meta-analysis of prevalence. J Epidemiol Community Health 2013;67:974-8.

26. Deeks JJ, Higgins PTJ, Altman D. Cochrane Handbook: General Methods For Cochrane Reviews: Ch 9: Analysing data and undertaking meta-analyses. Cochrane Handbook for Systematic Reviews of Interventions, 2011:243-96.

27. Moher D, Shamseer L, Clarke M, et al. Preferred reporting items for systematic review and meta-analysis protocols (PRISMA-P) 2015 statement. Syst Rev 2015;4:1-9. 